\title{
Erratum to: Hopf bifurcations through delay in pilot reaction in a longitudinal flight
}

\author{
A. Halanay • A. Ioniţă • C.-A. Safta
}

Published online: 3 October 2012

(C) Springer Science+Business Media Dordrecht 2012

\section{Erratum to: Nonlinear Dyn (2010) 60:413-423 DOI 10.1007/s11071-009-9605-x}

Following is the correct form of the formula (4.11) in the original paper

$$
\begin{aligned}
\langle\psi, \varphi\rangle= & \sum_{j=1}^{3} \bar{\psi}_{j}(0) \varphi_{j}(0)+\int_{-\mu-\tau_{c}}^{0}\left[b_{1} k \bar{\psi}_{1}\left(\xi+\tau_{c}+\mu\right)\right. \\
& \left.+b_{2} k \bar{\psi}_{2}\left(\xi+\tau_{c}+\mu\right)\right] \varphi_{3}(\xi) d \xi
\end{aligned}
$$

The online version of the original article can be found under doi:10.1007/s11071-009-9605-x.

\section{A. Halanay $(\bowtie)$}

Department of Mathematics I,

University Politehnica of Bucharest,

313 Splaiul Independentei, 060042, Bucharest, Romania e-mail: halanay@mathem.pub.ro

\footnotetext{
A. Ioniţă

Institute for Theoretical and Experimental Analysis of Aeronautical Structures, 220 Bd. Iuliu Maniu, 061126, Bucharest, Romania

e-mail: achim.ionita@straero.ro

C.-A. Safta

Department of Hydraulics and Hydraulic Machinery, University Politehnica of Bucharest, 313 Splaiul Independenţei, 060042, Bucharest, Romania e-mail: carmen123safta@yahoo.com
}

Consequently, formula (4.13) becomes

$$
\bar{d}=\left[\sum_{j=1}^{3} \gamma_{j} \overline{\tilde{\gamma}}_{j}+\gamma_{3} k\left(b_{1} \overline{\tilde{\gamma}}_{1}+b_{2} \overline{\tilde{\gamma}}_{2}\right) \tau_{c} e^{-i \nu_{0} \tau_{c}}\right]^{-1} .
$$

